\title{
A CLASS OF FUNCTIONS AND THEIR DEGREE OF APPROXIMATION BY ALMOST $(N, p, \alpha)$ METHOD
}

\author{
RAJIV SINHA AND HEMANT KUMAR
}

\begin{abstract}
Qureshi [6] proved a theorem for the degree of approximation of a periodic function $\bar{f}$, conjugate to a $2 \pi$-periodic function $f$ and belonging to the class Lip $\theta$, by almost matrix mean of its conjugate series. The above theorem was further generalized by Qureshi and Nema [8] for a function belonging to the class $W\left(L^{p}, \Psi_{1}(t)\right)$ by almost matrix mean. In the present paper we have discussed degree of approximation of above class of functions by almost $(N, p, \alpha)$ method.
\end{abstract}

1. Let $f(x)$ be periodic with period $2 \pi$ and integrable in the sense of Lebesgue. The Fourier series associated with $f(x)$, is given by

$$
f(x) \sim \frac{a_{0}}{2}+\sum_{n=1}^{\infty}\left(a_{n} \cos n x+b_{n} \sin n x\right)
$$

then

$$
\bar{f}(x) \sim \sum_{n=1}^{\infty}\left(b_{n} \cos n x-a_{n} \sin n x\right),
$$

is called the conjugate series of $f(x)$.

A function $f \in \operatorname{Lip} \theta$ if

$$
f(x+h)-f(x)=O\left(|h|^{\theta}\right) \text { for } \quad 0<\theta \leq 1,
$$

We define the norm $\|\cdot\|_{p}$ by

$$
\|f\|_{p}=\left(\int_{0}^{2 \pi}|f(x)|^{p} d x\right)^{1 / p}, \quad p \geq 1
$$

and the degree of approximation $E_{n}(f)$ by

$$
E_{n}(f)=\min _{T_{n}^{\prime}}\left\|f-T_{n}\right\|_{p} \quad \text { (see Zygmund [7]) }
$$

where $T_{n}$ is a trigonometrical polynomial of degree $n$. We say

$$
f(x) \in \operatorname{Lip}(\theta, q) \text { for } a \leq x \leq b
$$

Received November 15, 1995.

Key words and phrases. Fourier series, almost matrix means, almost $(N, p, \alpha)$ mean, almost Nörlund means. 
if

$$
\left(\int_{a}^{b}|f(x+h)-f(x)|^{q} d x\right)^{1 / q} \leq A|h|^{\theta}, \quad 0 \leq \theta<1, q \geq 1,
$$

where $A$ is some constant. (see def. 5.38 of McFadden [2])

Given a positive increasing function $\Psi_{1}(t)$ and an integer $p>1$, we notice (see Qureshi [4]) $f(x) \in \operatorname{Lip}\left(\Psi_{1}(t), p\right)$ if.

$$
\left(\int_{0}^{2 \pi}|f(x+t)-f(x)|^{p} d x\right)^{1 / p}=O\left(\Psi_{1}(t)\right)
$$

and that $f(x) \in W\left(L^{p}, \Psi_{1}(t)\right)$ if

$$
\left(\int_{0}^{2 \pi}|f(x+t)-f(x)|^{p} \sin ^{\beta p} x d x\right)^{\frac{1}{p}}=O\left(\Psi_{1}(t)\right), \quad \beta \geq 0 .
$$

In case $\beta=0$, we find that our newly defined class $W\left(L^{p}, \Psi_{1}(t)\right)$ coincides with the class $\operatorname{Lip}\left(\Psi_{1}(t), p\right)$.

Lorentz [1] has defined:

Definition 1. A sequence $\left\{S_{n}\right\}$ is said to be almost convergent to a limit $S$, if

$$
\lim _{n \rightarrow \infty} \frac{1}{n+1} \sum_{k=p}^{n+p} S_{k}=S
$$

uniformly with respect to $p$.

An almost convergence is a generalization of ordinary convergence. Qureshi [5] defined almost Nörlund means. Qureshi [3] have also defined almost triangular matrix means as:

Definition 2. If $\left(a_{n, k}\right)(n=0,1, \ldots, K=0,1, \ldots n) ; a_{n, 0}=1$ be a triangular matrix with real or complex elements, then a series $\sum_{n=0}^{\infty} u_{n}$ with the sequence of partial sums $\left\{S_{n}\right\}$ is said to be almost triangular matrix summable to $S$, provided

$$
\sigma_{n, p}=\sum_{k=0}^{n} a_{n, k} S_{k, p} \rightarrow S \quad \text { as } \quad n \rightarrow \infty
$$

uniformly with respect to $p$, where

$$
S_{k, p}=\frac{1}{K+1} \sum_{\mu=p}^{k+p} S \mu
$$

Definition 3. In above definition almost matrix summability reduces to almost $(N, p, \alpha)$ summability if

$$
a_{n, k}=\frac{a_{k} p_{n-k}}{(p \times \alpha)_{n}}=\frac{a_{k} p_{n-k}}{r_{n}}
$$


where

$$
\begin{aligned}
r_{n}=(p \times \alpha)_{n} & =p_{0} \alpha_{n}+p_{1} \alpha_{n-1}+\cdots+p_{n} \alpha_{0} \\
& =\sum_{k=0}^{n} \alpha_{k} p_{n-k} .
\end{aligned}
$$

Now the series $\sum_{n=0}^{\infty} u_{n}$ with the sequence of partial sums $\left\{S_{n}\right\}$ is said to be almost $(N, p, \alpha)$ summable to $S$, provided

$$
\tau_{n, p}=\sum_{k=0}^{n} \frac{\alpha_{k} p_{n-k} S_{k, p}}{(p \times \alpha)_{k}}=\frac{1}{(p \times \alpha)_{n}} \sum_{k=0}^{n} \alpha_{k} p_{n-k} S_{k, p}
$$

uniformly with respect to $p$, where

$$
S_{k, p}=\frac{1}{K+1} \sum_{\mu=p}^{k+p} S_{\mu} .
$$

2. Qureshi [6] proved the following theorems.

Theorem $\mathbb{A}$. The degree of approximation of a periodic function $\bar{f}$, conjugate to a $2 \pi$ periodic function $f$ and belonging to the class of Lip $\theta$ by almost Nörlund means of its conjugate series, is given by

$$
\left|\bar{f}(x)-\bar{t}_{n, p}(x)\right|=\left\{\begin{array}{l}
O\left[\left(\frac{1}{n}\right)^{\theta}\right] ; \quad 0<\theta<1 \\
O\left[\left(\frac{1}{n} \log n\right)\right] ; \quad \theta=1
\end{array}\right.
$$

where $\bar{t}_{n, p}(x)$ are the almost Nörlund means of the series (1.2) and the sequence $\left\{p_{n}\right\}$ is non-negative and non-increasing such that

$$
\sum_{k=0}^{n} \frac{p_{n-k}}{K+1}=O\left(\frac{P_{n}}{n}\right)
$$

Theorem $\mathbb{B}$. If a function $\overline{f(x)}$ is conjugate to a $2 \pi$ periodic function $f(x)$ belonging to the class of Lip $\theta$ for $0<\theta \leq 1$, then

$$
\left|\bar{\sigma}_{n, p}-\overline{f(x)}\right|=\left\{\begin{array}{l}
O\left[\left(\frac{1}{n}\right)^{\theta-1} \sum_{k=0}^{n} \frac{a_{n, k}}{K+1}\right] ; 0<\theta<1 \\
O\left[(\log n) \sum_{k=0}^{n} \frac{a_{n, k}}{K+1}\right] ; \theta=1
\end{array}\right.
$$

where $\bar{\sigma}_{n, p}$ are the almost triangular matrix means of the series (1.2). 
The above theorems were further generalized by Qureshi and Nema [8] for a periodic function $f(x)$ and belonging to the class $W\left(L^{p}, \Psi_{1}(t)\right)$.

However our theorem is as follows:

Theorem. If $f(x)$ is periodic and belongs to the class $W\left(L^{p}, \Psi_{1}(t)\right)$, then

$$
\left\|\bar{\tau}_{n, p}-\bar{f}\right\|=O\left[\frac{\Psi_{i}\left(\frac{1}{n}\right)(n)^{\beta+1+\left(\frac{1}{p}\right)}}{(p \times \alpha)_{n}} \sum_{k=0}^{n} \frac{\alpha_{k} p_{n-k}}{K+1}\right]
$$

provided $\Psi_{1}(t)$ satisfies the following conditions:

$$
\begin{aligned}
& \text { (a) }\left(\int_{0}^{\pi / n}\left(\frac{t|\Psi(t)|^{p}}{\Psi_{1(t)}}\right)^{p} \sin ^{\beta p} t d t\right)^{1 / p}=O\left(\frac{1}{n}\right) \\
& \text { (b) }\left(\int_{\frac{\pi}{n}}^{\pi}\left(\frac{t^{-\delta}|\Psi(t)|}{\Psi_{1(t)}}\right)^{p} d t\right)^{1 / p}=O\left(n^{\delta}\right)
\end{aligned}
$$

where $\delta$ is an arbitrary number such that $q(1-\delta)-1>0$, condition $(a)$ and $(b)$ hold uniformly in $x$,

$$
\Psi(t)=f(x+t)-f(x-t),
$$

$\overline{f(x)}$ is a function conjugate to $f, \bar{\tau}_{n, p}$ are almost $(N, p, \alpha)$ means of the series (1.2) and $\left\{\frac{\alpha_{k} p_{n-k}}{r_{n}}\right\}_{k=0}^{n}$ is a non-negative sequence with respect to $K$.

\section{Proof of the Theorem.}

Let $\bar{S}_{k}$ be the $k$-th partial sum of the conjugate series (1.2). Then we have

$$
\bar{S}_{k}(x)-\bar{f}(x)=-\frac{1}{\pi} \int_{0}^{\pi} \Psi(t) \frac{\cos \left(K+\frac{1}{2}\right) t}{2 \sin \frac{t}{2}} d t
$$

and

$$
\begin{aligned}
\bar{S}_{k, p}-f(x) & =\frac{1}{K+1} \sum_{\mu=p}^{k+p}\left(\bar{S}_{\mu}(x)-\bar{f}(x)\right) \\
& =-\frac{1}{2 \pi(K+1)} \int_{0}^{\pi} \Psi(t) \sum_{\mu=p}^{k+p} \frac{\cos \left(\mu+\frac{1}{2}\right)^{t}}{\sin \frac{t}{2}} d t \\
& =\frac{1}{2 \pi(K+1)} \int_{0}^{\pi} \Psi(t) \frac{\sin p t-\sin (K+p+1) t}{2 \sin ^{2} \frac{t}{2}} d t
\end{aligned}
$$

Now we have :

$$
\bar{\tau}_{n, p}-\bar{f}(x)=\sum_{k=0}^{n} \frac{\alpha_{k} p_{n-k}}{(p \times \alpha)_{k}}\left(\bar{S}_{k, p}(x)-\bar{f}(x)\right)
$$




$$
\begin{aligned}
& =\frac{1}{(p \times \alpha)_{n}} \sum_{k=0}^{n} \alpha_{k} p_{n-k}\left(\bar{S}_{k, p}(x)-\bar{f}(x)\right) \\
& =\frac{1}{2 \pi} \int_{0}^{\pi} \frac{\Psi(t)}{(p \times \alpha)_{n}} \sum_{K=0}^{n} \frac{\alpha_{k} p_{n-k}}{(K+1)} \frac{\cos (K+2 p+1) t / 2 \sin (K+1) t / 2}{\sin ^{2} t / 2} d t \\
& =\frac{1}{2 \pi}\left[\int_{0}^{\pi / n}+\int_{\pi / n}^{\pi}\right] \frac{\Psi(t)}{(p \times \alpha)_{n}} \sum_{k=0}^{n} \frac{\alpha_{k} p_{n-k}}{(K+1)} \frac{\cos (K+2 p+1) t / 2 \sin (K+1) t / 2}{\sin ^{2} t / 2} d t \\
& =I_{1}+I_{2}, \quad \text { say. }
\end{aligned}
$$

Now

$$
I_{1}=\frac{1}{2 \pi} \int_{0}^{\pi / n} \frac{\Psi(t)}{(p \times \alpha)_{n}} \sum_{k=0}^{n} \frac{\alpha_{k} p_{n-k}}{(k+1)} \frac{\cos (K+2 p+1) t / 2 \sin (K+1) t / 2}{\sin ^{2} t / 2} d t
$$

Applying Holder's inequality and the fact $\Psi(t) \in W\left(L^{p}, \Psi_{1}(t)\right)$, we have

$$
\begin{aligned}
I_{1} \leq & \frac{1}{2 \pi}\left(\int_{0}^{\pi / n}\left(\left(\frac{t \mid \Psi(t)}{\Psi_{1}(t)}\right) \sin ^{\beta} t\right)^{p} d t\right)^{1 / p} \\
& \times\left(\int_{0}^{\pi / n}\left(\frac{\Psi_{1}(t)}{(p \times \alpha)_{n} t}\left|\sum_{k=0}^{n} \frac{\alpha_{k} p_{n-k}}{(K+1)} \frac{\cos (K+2 p+1) t / 2 \sin (K+1) t / 2}{\sin ^{2} t / 2 \sin ^{\beta} t}\right|\right)^{q} d t\right)^{1 / q} \\
= & O\left[\left(\frac{1}{n}\right)\right] O\left[\left(\left(\int_{0}^{\pi / n} \frac{\Psi_{1}(t)}{(p \times \alpha)_{n} t} \sum_{k=0}^{n} \frac{\alpha_{k} p_{n-k}}{(K+1)} \frac{(K+1)|\sin t / 2|}{\sin ^{2} t / 2 \sin ^{\beta} t}\right)^{q} d t\right)^{1 / q}\right] \text { by condition (a). } \\
= & O\left[\left(\frac{1}{n}\right)\right] O\left[\frac{1}{(p \times \alpha)_{n}} \sum_{k=0}^{n} \alpha_{k} p_{n-k}\left(\int_{0}^{\pi / n}\left(\frac{\Psi_{1}(t)}{t^{\beta+2}}\right)^{q} d t\right)^{1 / q}\right],
\end{aligned}
$$

applying mean-value theorem, we have

$$
\begin{aligned}
I_{1} & =O\left[\left(\frac{1}{n}\right)\right] O\left[\frac{1}{(p \times \alpha)_{n}} \sum_{k=0}^{n} \alpha_{k} p_{n-k}\left(\Psi_{1}^{2}\left(\frac{\pi}{n}\right) \int_{1}^{\pi / n} \frac{d t}{t^{(2+\beta) q}}\right)^{1 / q}\right] \\
& =O\left[\left(\frac{1}{n}\right)\right] O\left[\frac{1}{(p \times \alpha)_{n}} \sum_{k=0}^{n} \alpha_{k} p_{n-k}\left(\left(\Psi_{1}\left(\frac{\pi}{n}\right)^{q} \frac{t^{-(2+\beta) q+1}}{-(2+\beta) q+1}\right)_{1}^{\pi / n}\right)^{1 / q}\right] \\
& =O\left[\left(\frac{1}{n}\right)\right] O\left[\frac{1}{(p \times \alpha)_{n}} \sum_{k=0}^{n} \alpha_{k} p_{n-k} \Psi_{1}\left(\frac{\pi}{n}\right)(n)^{\beta+1+(1 / p)}\right] \\
& =O\left[\frac{\Psi_{1}\left(\frac{1}{n}\right)(n)^{\beta+1+(1 / p)}}{(p \times \alpha)_{n}} \sum_{k=0}^{n} \frac{\alpha_{k} p_{n-k}}{(K+1)}\right] .
\end{aligned}
$$

Since we have:

$$
\frac{\Psi_{1}\left(\frac{1}{n}\right)(n)^{\beta+1+(1 / p)}}{n(p \times \alpha)_{n}} \sum_{k=0}^{n} \alpha_{k} p_{n-k}<\frac{\Psi_{1}\left(\frac{1}{n}\right)(n)^{\beta+1+(1 / p)}}{(p \times \alpha)_{n}} \sum_{k=0}^{n} \frac{\alpha_{k} p_{n-k}}{(K+1)}
$$


where $\frac{1}{p}+\frac{1}{q}=1$ such that $1 \leq p \leq \infty$.

Also, similarly as above

$$
\begin{aligned}
I_{2} \leq & \left(\int_{\pi / n}^{\pi}\left|\frac{t^{-\delta} \sin ^{\beta} t \Psi(t)}{\Psi_{1}(t)}\right|^{p} d t\right)^{1 / p} \\
& \times\left(\int_{\pi / n}^{\pi}\left|\frac{1}{(p \times n)_{n}} \sum_{k=0}^{n} \frac{\alpha_{k} p_{n-k}}{(K+1)} \cdot \frac{\cos (K+2 p+1) t / 2 \sin (K+1) t / 2 \Psi_{1}(t)}{t^{-\delta+2} \sin ^{\beta} t}\right|^{q} d t\right)^{1 / q} \\
= & O\left[\left(\int_{\pi / n}^{\pi}\left(t^{-\delta} \frac{|\Psi(t)|}{\Psi_{1}(t)}\right) d t\right)^{1 / p}\right] O\left[\frac{1}{\sin ^{\beta}\left(\frac{1}{n}\right)}\right] \\
& \times O\left[\frac { 1 } { ( p \times \alpha ) _ { n } } \sum _ { k = 0 } ^ { n } \frac { \alpha _ { k } p _ { n - k } } { ( K + 1 ) } \left(\int_{\pi / n}^{\pi} \frac{\left.\left.\left(\frac{\Psi_{1}(t)}{t^{-\delta+2}}\right)^{q} d t\right)^{1 / q}\right]}{=}\right.\right. \\
= & O\left[(n)^{\delta}\right] O\left[\frac{1}{\left(\frac{1}{n}\right)^{\beta}}\right] O\left[\frac{1}{(p \times \alpha)_{n}} \sum_{k=0}^{n} \frac{\alpha_{k} p_{n-k}}{(K+1)}\left(\int_{1}^{n}\left(\frac{\Psi_{1}(1 / y)}{y^{\delta-2}}\right) \frac{d y}{y^{2}}\right)^{1 / q}\right] \text { by condition (b) } \\
= & O\left[(n)^{\delta}\right] O\left[(n)^{\beta}\right] O\left[\frac{\Psi_{1}\left(\frac{1}{n}\right)}{(p \times \alpha)_{n}} \sum_{k=0}^{n} \frac{\alpha_{k} p_{n-k}(n)^{-\delta+2-(1 / q)}}{(K+1)}\right] O\left[\frac{\Psi_{1}\left(\frac{1}{n}\right)}{(p \times \alpha)_{n}} \sum_{k=0}^{n} \frac{\alpha_{k} p_{n-k}}{(K+1)}\left(\int_{1}^{n} \frac{d y}{y^{\delta q-2 q+2}}\right)^{1 / q}\right] \\
= & O\left[\frac{\Psi_{1}\left(\frac{1}{n}\right)(n)^{\beta+2-(1 / q)}}{(p \times \alpha)_{n}} \sum_{k=0}^{n} \frac{\alpha_{k} p_{n-k}}{(K+1)}\right] .
\end{aligned}
$$

Hence

$$
\left|\bar{\tau}_{n, p}(x)-\overline{f(x)}\right|=O\left[\frac{\Psi_{1}\left(\frac{1}{n}\right)(n)^{\beta+1+(1 / p)}}{(p \times \alpha)_{n}} \sum_{k=0}^{n} \frac{\alpha_{k} p_{n-k}}{(K+1)}\right]
$$

uniformly for $x$, therefore,

$$
\left\|\bar{\tau}_{n, p}(x)-\overline{f(x)}\right\|=\sup _{0 \leq x \leq 2 \pi}\left|\bar{\tau}_{n, p}(x)-\overline{f(x)}\right|=O\left[\frac{\Psi_{1}\left(\frac{1}{n}\right)(n)^{\beta+1+(1 / p)}}{(p \times \alpha)_{n}} \sum_{k=0}^{n} \frac{\alpha_{k} p_{n-k}}{(K+1)}\right]
$$

This complete the proof of the theorem.

The following Corollaries can be derived from the theorem:

Corollary 1. If $\beta=0$ and $\Psi_{1}(t)=t^{\gamma}$, then the degree of approximation of a function $f(x)$, conjugate to a $2 \pi$-periodic function $f$ belong to the class $\operatorname{Lip}(\gamma, p), 0<\gamma \leq 1$, is given by:

$$
\left\|\bar{\tau}_{n, p}(x)-\overline{f(x)}\right\|=O\left[\frac{\left(\frac{1}{n}\right)^{\gamma-1-(1 / p)}}{(p \times \alpha)_{n}} \sum_{k=0}^{n} \frac{\alpha_{k} p_{n-k}}{(K+1)}\right]
$$

where $\bar{\tau}_{n, p}(x)$ are almost $(N, p, \alpha)$ means of the series (1.2) and $\left\{\frac{\alpha_{k} p_{n-k}}{(p \times \alpha)_{n}}\right\}$ is non-negative sequence with respect to $K$. 
Proof. Since

$$
\begin{aligned}
\left|\bar{\tau}_{n, p}(x)-\overline{f(x)}\right| & =O\left[\frac{\Psi_{1}\left(\frac{1}{n}\right)(n)^{\beta+1+(1 / p)}}{(p \times \alpha)_{n}} \sum_{k=0}^{n} \frac{\alpha_{k} p_{n-k}}{(K+1)}\right] \\
& =O\left[\frac{\left(\frac{1}{n}\right)^{\gamma}(n)^{1+(1 / p)}}{(p \times \alpha)_{n}} \sum_{k=0}^{n} \frac{\alpha_{k} p_{n-k}}{(K+1)}\right] \\
& =O\left[\frac{\left(\frac{1}{n}\right)^{\gamma-1-(1 / p)}}{(p \times \alpha)_{n}} \sum_{k=0}^{n} \frac{\alpha_{k} p_{n-k}}{(K+1)}\right]
\end{aligned}
$$

which completes the proof.

Corollary 2. If $p \rightarrow \infty$ in Corollary 1, then

$$
\left\|\bar{\tau}_{n, p}(x)-\overline{f(x)}\right\|=O\left[\frac{\left(\frac{1}{n}\right)^{\gamma-1}}{(p \times \alpha)_{n}} \sum_{k=0}^{n} \frac{\alpha_{k} p_{n-k}}{(K+1)}\right]
$$

and we have theorem $B$, and if further $\alpha=1$, we have theorem $A$ for $0<\alpha<1$. The proof of Corollary 2 is obvious.

\section{References}

[1] G. G. Lorentz, "A contribution to the theory of divergent series," Acta Match., 80 (1948), 167-190.

[2] L. Mc Fadden, "Absolute Nörlund summability," Duke Math. J., 9(1942), 163-207.

[3] K. Qureshi, "On the degree of approximation of functions belonging to the class $\operatorname{Lip}(\alpha, q)$, Tamkang Jour. Math., 15(1)(1984), 5-11.

[4] K. Qureshi, "On the degree of approximation of function belonging to weighted $W\left(L^{p}, \Psi_{1}(t)\right)$ class," Indian J. Pure Appl. Math., 13(4)(1982), 471-475.

[5] K. Qureshi, "On the degree of approximation of a periodic function $f$ by almost Nörlund means," Tamkang J. Math., 12(1)(1961), 35-36.

[6] K. Qureshi, "On the degree of approximation of a class of functions by means of a conjugate series," R.U.M.J., 15(1984), 63-68.

[7] A. Zygmund, Trigonometric series, Vol. 1, 2nd Ed. Cambridge University Press, Cambridge, 1959.

[8] K. Qureshi and H. K. Nema, "A class of functions and their degree of approximation," The Mathematics Student, 52(1-4)(1984), 33-40.

Department of Mathematics, S.M. College, Chandausi (U.P.), PIN: 202412, INDIA.

Department of Physics, S.M. College, Chandausi (U.P.), PIN: 202412, INDIA. 